\title{
Zur Rolle der NGOs in der Entwicklungspolitik der Schweiz
}

Joseph Deiss and Walter Fust

\section{OpenEdition}

1 Journals

\section{Electronic version}

URL: http://journals.openedition.org/sjep/503

DOI: 10.4000/sjep.503

ISSN: 1663-9677

\section{Publisher}

Institut de hautes études internationales et du développement

\section{Printed version}

Date of publication: 1 novembre 2004

Number of pages: 125-132

ISBN: 2-88247-057-6

ISSN: 1660-5926

\section{Electronic reference}

Joseph Deiss und Walter Fust, "Zur Rolle der NGOs in der Entwicklungspolitik der Schweiz » Schweizerisches Jahrbuch für Entwicklungspolitik [Online], 23-2 | 2004, Online erschienen am: 10 Juni 2010, abgerufen am 07 September 2020. URL : http://journals.openedition.org/sjep/503 ; DOI : https://doi.org/10.4000/sjep.503 


\title{
Zur Rolle der NGOs in der Entwicklungspolitik der Schweiz ${ }^{1}$
}

\author{
Joseph Deiss* und Walter Fust**
}

\section{Vorwort}

Der gesellschaftliche Wandel, die zunehmende internationale Verflechtung und die Globalisierung verleihen den zivilgesellschaftlichen Organisationen eine wachsende Bedeutung. Auf der Bühne der Aussenpolitik spielen nicht-staatliche Akteure, darunter vor allem die „Non-Governmental Organizations“ (NGOs), eine immer wichtigere Rolle. Dies gilt auch für die Entwicklungszusammenarbeit und die humanitäre Hilfe. Doch gerade auf diesem Gebiet haben das Wirken privater schweizerischer Organisationen und ihre Zusammenarbeit mit dem Staat bereits eine jahrzehntealte Tradition. NGOs setzen sich oft für Themen und Anliegen ein, die keine eigene Lobby haben und deshalb auf der politischen Agenda keinen zentralen Stellenwert einnehmen. Dies war der Ausgangspunkt für die Gründung von privaten Hilfswerken und ist noch heute eine der Stärken der Entwicklungsorganisationen.

Ein Blick auf die Ursprünge der schweizerischen Entwicklungszusammenarbeit

„Werfen wir einen Blick auf die Gesamtheit der schweizerischen Entwicklungshilfe, so dürfen wir mit Freuden feststellen, dass sie heute sowohl vom Staat wie von den Privaten als eine selbstverständliche Notwendigkeit betrachtet wird. Sie bildet einen wesentlichen Teil der schweizerischen Aussenpolitik“.

Mit diesen Worten zog Frau Regina Kägi-Fuchsmann, eine Pionierin der privaten schweizerischen Entwicklungszusammenarbeit, im Jahr 1964 Bilanz. Frau KägiFuchsmann war langjährige Leiterin des Schweizerischen Arbeiterhilfswerkes (1934-1951). Sie gehörte zu jenem privaten Personenkreis, der sich in den 50er Jahren - überzeugt von der Notwendigkeit einer schweizerischen Entwicklungshilfe als nationale Aufgabe - unermüdlich für die Schaffung einer nicht-staatlichen Trägerschaft einsetzte. Diese Bemühungen resultierten 1955 in der Gründung eines politisch und konfessionell neutralen Vereins - des Schweizerischen Hilfswerks für aussereuropäische Gebiete (SHAG, ab 1965 „Helvetas“).

Die Förderer des Schweizerischen Hilfswerks für aussereuropäische Gebiete begründeten ihre Initiative damit,

1 Der Artikel von Joseph Deiss und Walter Fust : „Zur Rolle der NGOs in der Entwicklungspolitik der Schweiz" erschien im Jahr 2003 in Österreich in Öffentliche Verwaltung und Nonprofit-Organisationen, Festschrift für Reinbert Schauer, herausgegeben von Ernst-Bernd Blümle, Helmut Pernsteiner, Robert Purtschert, René Clemens Andessner, Linde Verlag, Wien, 2003, Seite 104-114. Wir danken dem Linde Verlag für die Abdrucksbewilligung.

* Bundesrat, Vorsteher des Eidgenössischen Volkswirtschaftsdepartements.

** Direktor der Direktion für Entwicklung und Zusammenarbeit (DEZA). 
口 ,dass die Art und das Ausmass der den wirtschaftlich unterentwickelten Ländern geleisteten Hilfe für das Schicksal der ganzen Welt entscheidende Bedeutung hat" und

$\square$ „,dass es für das Schweizervolk notwendig sei, dass es mit der Wirklichkeit der Weltsituation, vor allem mit der engen Verflechtung aller Kontinente in wirtschaftlicher, sozialer und kultureller Beziehung vertraut gemacht und zur solidarischen Hilfe aufgerufen werde".

Die private schweizerische Entwicklungshilfe entstand in den 50er Jahren auch auf der Basis der schweizerischen Hilfe für das kriegszerstörte Europa. Caritas, das Hilfswerk der Evangelischen Kirchen der Schweiz, das Schweizerische Arbeiterhilfswerk, das Schweizerische Rote Kreuz und Swissaid waren führende Träger der Europahilfe. Diese Organisationen entwickelten sich in den 60er Jahren zu wichtigen Akteuren der privaten Entwicklungszusammenarbeit.

Ein weiterer Impuls für das Erstarken der nicht-staatlichen Entwicklungshilfe ging von der Privatwirtschaft aus: Sie ist die Trägerin der 1959 gegründeten Schweizerischen Stiftung für Technische Entwicklungshilfe (später „Swisscontact“).

Als Vorläufer der privaten Entwicklungszusammenarbeit sind schliesslich auch die schweizerischen Missionswerke zu erwähnen.

Das Heranwachsen von privaten und nicht-profitorientierten Organisationen als Förderer und Träger der schweizerischen Entwicklungszusammenarbeit ist nicht einfach als logische Ableitung aus der humanitären Tradition der Schweiz zu verstehen. Es handelt sich vielmehr um das Resultat eines neuen Bewusstseins über die soziale und wirtschaftliche Lage in den Ländern des Südens - vor allem in den sich von der Kolonialherrschaft emanzipierenden Nationen. Die sozioökonomische Situation in diesen Ländern wurde erstmals als ein internationales Problem und als eine Bedrohung für den Frieden in der Welt wahrgenommen.

\section{Die gesellschaftliche Verankerung der NGOs}

Ein wichtiges Merkmal der privaten Entwicklungsorganisationen war von Anfang an ihre tiefe Verankerung in der schweizerischen Gesellschaft. Die gesellschaftliche Abstützung ruht unter anderem auf folgenden Pfeilern:

- Mobilisierung von Mitgliedern und Lokalgruppen (Schweizerisches Hilfswerk für aussereuropäische Gebiete, heute Helvetas);

$\square$ Engagement kirchlicher Kreise, erwachsend aus der Europahilfe (HEKS, Caritas) oder aus der katholischen Laienbewegung (Fastenopfer);

- Engagement der Arbeiterorganisationen, aufbauend auf der Tradition der europäischen Arbeiter- und Kinderhilfe (Schweizerisches Arbeiterhilfswerk);

$\checkmark$ privatwirtschaftliches Engagement (Swisscontact).

Mit diesen gesellschaftlichen Wurzeln bilden die Nicht-Regierungsorganisationen (NGOs) eine wichtige innenpolitische Basis für die schweizerische Entwicklungszusammenarbeit.

Die schweizerische Regierung, der Bundesrat, stand den Trägern der privaten Entwicklungshilfe von Anfang an wohlwollend und fördernd gegenüber. Ebenso 
beflügelte die Vorreiterrolle der privaten Organisationen das rasch wachsende staatliche Engagement. Im Jahre 1961 wurde im Aussenministerium, dem Politischen Departement (heute Eidgenössisches Departement für auswärtige Angelegenheiten EDA), der Dienst für technische Zusammenarbeit eingerichtet, aus dem im Laufe der folgenden Jahrzehnte die Direktion für Entwicklung und Zusammenarbeit (DEZA) heranwuchs. Im selben Jahr gewährte das Parlament einen ersten Entwicklungshilfe-Kredit von 60 Millionen Franken. So entstanden die organisatorischen und materiellen Voraussetzungen für die enge Zusammenarbeit und Partnerschaft zwischen staatlichen und privaten Akteuren.

Die Rolle der NGOs ist auch im Bundesgesetz über die internationale Entwicklungszusammenarbeit und humanitäre Hilfe vom 19. März 1976 fest verankert. Ebenso betonen die verschiedenen ans Parlament gerichteten Botschaften des Bundesrates über die Weiterführung der technischen Zusammenarbeit und der Finanzhilfe zugunsten von Entwicklungsländern die Bedeutung der Partnerschaft mit den NGOs, und zwar sowohl für den politischen Dialog und den Erfahrungsaustausch als auch auf der Ebene der praktischen Umsetzung der schweizerischen Entwicklungszusammenarbeit.

\section{Charakterisierung und Typologisierung der NGOs}

Das Spektrum der schweizerischen Entwicklungs-NGOs umfasst rund 30 grössere und 150 kleinere Organisationen mit unterschiedlichen thematischen und ideellen Ausrichtungen. Es reicht von professionell operierenden Hilfswerken bis hin zu weitgehend auf Freiwilligkeit beruhenden Solidaritätsgruppen. Einige dieser Organisationen haben eine nationale Ausstrahlung, während andere vorwiegend regional oder gar lokal verankert sind. Der Aktionsradius ist, je nach Organisation, weltumspannend oder geografisch eingeschränkt. Trotz dieser Diversität ist es möglich, eine grobe Karte der schweizerischen NGO-Landschaft im Entwicklungsbereich zu zeichnen.

Doch zuerst eine allgemeine Charakterisierung im Sinne einer idealtypischen Beschreibung. Was unterscheidet eine NGO von einem Privatunternehmen oder einer Beraterfirma? Zum einen die Tatsache, dass eine NGO nicht profitorientiert arbeitet und damit betriebswirtschaftlich anders organisiert ist. Darüber hinaus haben NGOs auch einen gesellschaftsgestalterischen Anspruch. Sie sind bestrebt, auf die Gestaltung von wirtschaftlichen und sozialen Strukturen einzuwirken.

Sie verstehen sich als Akteure gesellschaftspolitischer Prozesse. Darin liegt auch die entwicklungspolitische Bedeutung des Unterschiedes zwischen NGO und Privatfirma begründet.

Die schweizerischen privaten Entwicklungsorganisationen und Hilfswerke lassen sich grob wie folgt typologisieren:

1. Organisationen der Entwicklungspolitik: Die Arbeitsgemeinschaft der Hilfswerke und die Erklärung von Bern.

Diese Organisationen widmen sich der entwicklungspolitischen Analyse, der Informationsarbeit und der Bewusstseinsbildung. Sie stehen im Dialog mit Staat und Privatwirtschaft. Sie leisten Beiträge an die entwicklungspolitische Diskussion auf nationaler und internationaler Ebene. 
2. Spezialisierte Organisationen für operationelle Entwicklungszusammenarbeit: Helvetas, Swissaid, Swisscontact, Intercooperation.

Diese Organisationen führen umfassende Entwicklungsprogramme in Afrika, Asien und Lateinamerika durch. Sie nehmen in unterschiedlichem Masse Informations- und Sensibilisierungsaufgaben im Inland wahr.

3. Multifunktionale Hilfswerke mit einer spezialisierten Abteilung für operationelle Entwicklungszusammenarbeit und humanitäre Hilfe: Dazu gehören die kirchlichen Hilfswerke - unter ihnen Caritas und HEKS - sowie nichtkirchliche Organisationen, wie das Schweizerische Rote Kreuz und das Schweizerische Arbeiterhilfswerk.

Neben ihrem internationalen Engagement sind diese Organisationen auch in der Sozialarbeit in der Schweiz tätig. Sie übernehmen zum Teil ebenfalls Informations- und Sensibilisierungs-Arbeit im Inland.

4. Spezialisierte Organisationen für Kinderhilfe, wie z.B. Terre des Hommes. Diese Organisationen leisten humanitäre Hilfe und führen Entwicklungsprogramme in Asien, Afrika und Lateinamerika durch. Sie orientieren sich vornehmlich an der UNO-Kinderrechtskonvention.

5. Umwelt- und Naturschutzorganisationen, wie z.B. der WWF Schweiz oder Pro Natura. Diese Organisationen setzen sich für eine umweltverträgliche Entwicklung und für den Naturschutz in der Schweiz und im Ausland ein.

6. Organisationen für den Freiwilligen-Einsatz mit unterschiedlicher Ausrichtung, zusammengefasst in Unité. Diese Organisationen konzentrieren sich auf den Personaleinsatz in einem breiten Spektrum von Aktivitäten - von technisch-professionellen Unterstützungsprogrammen bis hin zur Förderung des interkulturellen Austausches und Dialoges.

Daneben gibt es zahlreiche kleinere, lokale Organisationen und Solidaritätsgruppen. Diese sind auf unterschiedlichste Art und Weise im humanitären, sozialen und entwicklungsbezogenen Bereich international tätig. In einigen Kantonen sind kantonale NGO-Föderationen entstanden, wie etwa in Genf, in der Waadt, im Jura und im Tessin. Sie fördern die operationelle Arbeit mit ihren Mitglied-Organisationen und den entwicklungspolitischen Dialog mit den lokalen Behörden.

Gemeinsam ist all diesen Organisationen, dass sie sich in ihrer Arbeit auf die Förderung einer nachhaltigen Entwicklung, die Bekämpfung von Ungerechtigkeit und Armut sowie die Beseitigung sozialer Missstände ausrichten.

Das von NGOs in der Schweiz mobilisierte Spendenpotenzial beläuft sich auf rund 250 Millionen Franken pro Jahr. Davon entfallen etwa 190 Millionen auf die Entwicklungsarbeit und 60 Millionen auf die humanitäre Hilfe.

Formen der Zusammenarbeit zwischen der DEZA und den schweizerischen NGOs

Operationelle Zusammenarbeit

Mit mehrjährigen Programmbeiträgen unterstützt die DEZA die Entwicklungsprogramme der 13 grösseren schweizerischen NGOs und NGO-Dachorganisa- 
tionen. Dabei formulieren die NGOs ihre programmatischen Ziele und Konzepte selber. Sie identifizieren ihre Partner in den Entwicklungsländern und tragen die Verantwortung für die Qualität der Programme. Die Beiträge sind an entsprechende finanzielle Eigenleistungen dieser Organisationen gebunden.

Diese staatlichen Zuschüsse sind keine Subventionen. Es sind verhandelte Beiträge an Entwicklungsprogramme mit klaren programmatischen Zielsetzungen. In diesem Sinne handelt es sich um Leistungsvereinbarungen.

Für die Beitragsperiode 2002-2004 werden sich die Programmbeiträge der DEZA auf durchschnittlich 60 Millionen Franken pro Jahr belaufen. Dies entspricht rund 14 Prozent des Budgets der DEZA für die bilaterale Entwicklungszusammenarbeit.

Kleinere NGOs mit ausgewiesenen Kapazitäten können sich für Einzelprojektbeiträge qualifizieren. Die jährlichen Aufwendungen für solche Zuschüsse belaufen sich auf drei bis vier Millionen Franken.

NGOs sind auch Partner für die Umsetzung von Entwicklungsprojekten des Bundes. In diesem Falle stehen die NGOs in einem Auftragsverhältnis zum Bund. Die wichtigsten Auftragnehmer sind Helvetas, Intercooperation und Swisscontact. Im Jahr 2000 haben schweizerische NGOs Aufträge der DEZA mit einem finanziellen Gesamtvolumen von 71 Millionen Franken realisiert. Andere Auftragnehmer sind Universitäten und spezialisierte Institute sowie private Beratungsfirmen.

Insgesamt wickelte die DEZA in der zweiten Hälfte der 90er Jahre rund 25 Prozent der bilateralen Entwicklungszusammenarbeit über schweizerische NGOs ab.

\section{Erfahrungsaustausch und politischer Dialog}

Zwischen den NGOs und der DEZA findet ein regelmässiger Dialog und Erfahrungsaustausch statt. Der Austausch über spezifische Themen von gemeinsamem Interesse dient der Förderung von Komplementaritäten und Synergien und einer laufenden Überprüfung der Angemessenheit von Strategien und Konzepten.

Auf der politischen Ebene leisten die NGOs dank ihrer operationellen Erfahrung und ihrer analytischen Grundlagenarbeit einen unerlässlichen Beitrag zu einer lebendigen entwicklungspolitischen Debatte und zur qualitativen Evolution der schweizerischen Entwicklungszusammenarbeit. Ins Gewicht fallen dabei vor allem ihre konstruktiv-kritischen Stellungnahmen zu wichtigen Fragen.

Beispielhaft für die politische Bedeutung der NGOs ist etwa ihre aktive Rolle zur Unterstützung des Beitritts der Schweiz zu den Bretton-Woods-Institutionen, zur WTO und zur UNO. Im Falle des UNO-Beitritts hat die Arbeitsgemeinschaft der Schweizerischen Hilfswerke die entsprechende Volksinitiative mitgetragen und einen wesentlichen Beitrag zur Unterschriftensammlung geleistet. Sie beteiligte sich auch aktiv an der Abstimmungskampagne, die am 3. März 2002 mit der Zustimmung des Schweizer Volkes zum UNO-Beitritt endete.

Zum politischen Dialog gehören aber auch kritische Stellungnahmen und Auseinandersetzungen. $\mathrm{Zu}$ den in der Öffentlichkeit umstrittenen Themen gehören z.B. die schweizerischen Positionen in der WTO, Handelsfragen allgemein, Patentrechte, Kriegsmaterialausfuhr, Exportrisikogarantie oder die internationale Rechtshilfe. 
Die eigenständige finanzielle Basis ist ein wesentliches Charakteristikum der Unabhängigkeit der NGOs. Beiträge und Aufträge der öffentlichen Hand (Bund, Kantone und Gemeinden) sind jedoch für die Einkünfte von NGOs wichtig geworden. Aufgrund der Diversität und Multifunktionalität der NGOs lässt sich aber kein einheitlicher und vergleichbarer Abhängigkeitsgrad von staatlicher Finanzierung berechnen.

Im Bereich der Entwicklungszusammenarbeit legt der Bund grosses Gewicht auf die finanziellen Eigenleistungen der NGOs. Die Programmbeiträge sollen nicht zu einem Instrument der Strukturerhaltung von NGOs in der Schweiz werden. Im Vordergrund müssen immer die entwicklungspolitischen und programmatischen Ziele stehen.

Zur Unabhängigkeit der NGOs gehört auch ihre eigenständige Stimme im politischen Dialog - das liegt im Wesen dieser Organisationen. Daraus ergibt sich ein Spannungspotenzial, sobald es um politisch heikle Themen oder Abstimmungsvorlagen geht. Die Unabhängigkeit der NGOs ist nicht allein eine Frage der Finanzierung, sondern auch eine Frage des demokratischen Freiraumes für divergierende politische Stellungnahmen. Die Pluralität der schweizerischen Gesellschaft soll sich auch in der Entwicklungspolitik niederschlagen können. Dazu gehört ein kritischer und verbindlicher entwicklungspolitischer Dialog. Wir müssen anerkennen, dass unser aussenpolitischer Anspruch, einen Beitrag zur Förderung der Menschenrechte, der Demokratie, der „Good Governance“ und zur Stärkung zivilgesellschaftlicher Strukturen zu leisten, nur dann glaubwürdig wirkt, wenn wir unsere eigenen zivilgesellschaftlichen Organisationen als kritische Mahner und Vorreiter, als manchmal auch unbequeme Partner in der politischen Entscheidfindung und als gesellschaftliche Interessenträger ernst nehmen.

Es gehört somit zur Unabhängigkeit der NGOs, dass sich diese kritisch in die politische Debatte einbringen.

\section{NGOs als Vorreiter und zivilgesellschaftliche Kraft}

Nicht-Regierungsorganisationen sind zu wichtigen Akteuren auf der internationalen Bühne geworden. Sie sind zunehmend international vernetzt. Sie spielen eine wachsende Rolle als Dialogpartner von multilateralen Organisationen sowie in der Auseinandersetzung um die globale Gouvernanz und internationale Regulierung.

In vielen Fällen haben NGOs und zivilgesellschaftliche Kräfte die Rolle von Vorreitern gespielt. Erwähnt seien hier zwei Beispiele:

- Im Rahmen der Internationalen Kampagne gegen Landminen war u.a. Handicap International eine der treibenden Kräfte. Die Kampagne wird heute von 1300 Organisationen in über 90 Ländern getragen und von einem aus mehreren NGOs bestehenden Komitee koordiniert. Die gemeinsamen Anstrengungen führten 1997 zur Verabschiedung der Konvention gegen die Verwendung, Lagerung, Herstellung und Proliferation von Anti-Personen-Minen. Von den 142 Signatarstaaten hatten bis Oktober 2001122 die Konvention ratifiziert (Schweiz 1998). Im Rahmen der Kampagne ist auch ein zweites Informa- 
tionsnetzwerk - abgestützt auf die Zivilgesellschaft - entstanden. Dies ermöglicht das Monitoring der Konvention und der ganzen Minenproblematik. Dank den NGOs ist auf diesem Gebiet eine Dynamik entstanden, welche die Regierungen aus eigener Kraft nicht hätten generieren können. Die Schweiz unterstützt das von Handicap International durchgeführte Opferhilfeprogramm in Südostasien, das aus der Kampagne heraus gewachsen ist.

ـ Ende der 80er Jahre lancierten Schweizer NGOs eine Entschuldungs-Kampagne. Die von 26 Schweizer Organisationen getragene Kampagne appellierte an den Bundesrat, alle Schulden der armen Länder zu annullieren. Eine Petition „Entwicklung braucht Entschuldung“ wurde von 250'000 Personen unterschrieben. Sie bewirkte, dass anlässlich der 700-Jahr-Feier der Eidgenossenschaft im Jahre 1991 der Rahmenkredit für Entwicklungszusammenarbeit durch ein eigentliches Entschuldungsprogramm ergänzt wurde. Dank dieser Initiative fanden Entschuldungsprogramme Eingang in die schweizerische Entwicklungszusammenarbeit. Das daraus entstehende aktive Engagement der Schweiz in der internationalen Entschuldungsdebatte trug wesentlich dazu bei, dass die Weltbank ihre Politik überprüfte und entsprechende Aktionen unternahm. Hier haben Schweizer NGOs also Pionierarbeit geleistet, deren Bedeutung weit über die schweizerische Entwicklungszusammenarbeit hinausreicht.

Diese Beispiele zeigen, dass wichtige Errungenschaften vielfach Resultat von zivilgesellschaftlichem Druck und von intensiven Prozessen der Auseinandersetzung sind.

Die Entwicklungspolitik braucht diese Auseinandersetzung. Sie braucht Partnerschaften mit der Zivilgesellschaft und dem Privatsektor. Sie braucht einen konstruktiv-kritischen Dialog zwischen Staat, Zivilgesellschaft und Privatsektor. Es braucht eine offene Debatte über entwicklungspolitische Probleme und strukturelle Zusammenhänge. Es braucht die gemeinsame Suche nach angepassten Lösungen für die anstehenden Herausforderungen. Nicht zuletzt auch deshalb, weil die globalen Entwicklungsprobleme heute die innenpolitische Agenda mitbestimmen - z.B. im Bereich der Umwelt- oder der Migrationsproblematik.

Eine lebendige NGO-Szene kann Wesentliches dazu beitragen, neuen Ansätzen und Lösungen Bahn zu brechen. In diesem Sinne ist es eine Priorität, dafür zu sorgen, dass etwa die Konfrontationen im Zuge der Globalisierungskritik - von Seattle über Davos bis nach Genua - nicht zu einer Polarisierung und Verhärtung der Fronten zwischen dem Staat und den NGOs führen. Vielmehr sollen sie zum Anlass für eine offene Auseinandersetzung über ihre tieferen Ursachen genommen werden. Diese Ereignisse deuten auch auf die Notwendigkeit hin, die Plattform des Dialoges zu erweitern, Allianzen zu stärken und die zivilgesellschaftlichen Organisationen als kritische, manchmal auch unbequeme Teilhaber in eine auf echter Partnerschaft basierende Zusammenarbeit einzubeziehen.

\section{Ausblick}

Die raschen Veränderungen im globalen Umfeld stellen die Entwicklungszusammenarbeit vor zusätzliche Herausforderungen. Neue soziale Gräben öffnen sich, und sozio-kulturelle Orientierungssysteme erodieren. Die Ausdehnung globaler Handlungsspielräume geht einher mit gesellschaftlichen Ohnmachtsgefühlen. 
Neue Formen des Protestes und der Opposition manifestieren sich. Das Potenzial für Extremismus nimmt zu. Das Ringen um die globalen öffentlichen Güter beispielsweise das Wasser - verschärft sich. Staat, Zivilgesellschaft und Privatwirtschaft sehen sich mit der Herausforderung konfrontiert, die Nachhaltigkeit der künftigen Entwicklung als gemeinsame Verantwortung zu verstehen.

Wirtschaftsstrukturen und Handel müsen so gestaltet werden, dass sie Armut verhindern. Eine gesunde Umwelt und der sorgsame Umgang mit den natürlichen Ressourcen sind Grundvoraussetzungen für eine tragfähige Entwicklung und dürfen nicht wirtschaftlichen Interessen untergeordnet werden. Gesellschaftlicher Ausgleich und Rechtsordnung müssen dazu beitragen, soziale und regionale Gegensätze zu überwinden und Gewalt zu verhindern. NGOs können einen wichtigen Beitrag leisten, auf die notwendigen politischen und wirtschaftlichen Veränderungen hinzuarbeiten, und zwar sowohl auf lokaler, regionaler wie auch auf globaler Ebene.

Die Förderung des konstruktiv-kritischen entwicklungspolitischen Dialoges und die aktive Zusammenarbeit mit den NGOs sind wesentliche Bestandteile der schweizerischen Entwicklungspolitik. Damit verbunden sind aber auch Anforderungen an die NGOs. Wir erwarten von den NGOs, dass sie

๖ sich im Rahmen der politischen Auseinandersetzung von Gewalt distanzieren;

๑ fundierte Analysearbeit liefern und machbare Alternativen aufzeigen können;

$\checkmark$ transparente Strukturen und rechenschaftspflichtige Führungs- und Managementmechanismen aufweisen;

$\square$ ihre Arbeit an Kriterien zielkonformer Wirkung messen und über ein funktionierendes Qualitätsmanagement verfügen.

NGOs sollen NGOs bleiben. Wir wollen keine GONGOs (,Government-organized Non-Governmental Organizations“, wir wollen zivilgesellschaftliche und qualifizierte Partner.

Abbildung 1: Private und öffentliche Entwicklungshilfe (EH) der Schweiz, 1995-2001 (in Millionen Schweizer Franken)

\begin{tabular}{|c|c|c|c|c|c|c|c|}
\hline & 1995 & 1996 & 1997 & 1998 & 1999 & 2000 & $2001^{p}$ \\
\hline Private Entwicklungshilfe & 219.3 & 226.6 & 225.7 & 250.0 & 275.4 & 285.4 & n.v.* \\
\hline \multicolumn{8}{|l|}{ Öffentliche Entwicklungshilfe } \\
\hline Bund & 1261.4 & 1250.5 & 1298.0 & 1279.9 & 1444.7 & 1479.9 & $1509^{p}$ \\
\hline davon bilaterale EH der DEZA & 488.6 & 479.4 & 489.1 & 488.1 & 465.6 & 472.8 & 502.0 \\
\hline $\begin{array}{l}\text { davon Aufträge und Beiträge an } \\
\text { Schweizer NGOs für bilaterale EH }\end{array}$ & 124.4 & 112.3 & 132.1 & 119.1 & 125.1 & 127.5 & $\begin{aligned} 128.1 \\
255 \%\end{aligned}$ \\
\hline Kantone & 11.2 & $\begin{aligned} 20.410 \\
9.6\end{aligned}$ & 20.910 & $\begin{aligned} 24.410 \\
13.2\end{aligned}$ & 20.010 & 27.010 & $\frac{13.6}{13.6}$ \\
\hline Gemeinden & 8.2 & 8.6 & 7.8 & 8.4 & 8.9 & 8.1 & 7.5 \\
\hline Total öffentliche Entwicklungshilfe & 1280.8 & 1268.7 & 1320.9 & 1301.5 & 1465.2 & 1502.1 & $1530^{\mathrm{p}}$ \\
\hline $\begin{array}{l}\text { Private Entwicklungshilfe in \% } \\
\text { der öffentlichen Entwicklungshilfe }\end{array}$ & 17.1 & 17.9 & 17.1 & 19.2 & 18.8 & 19.0 & n.v.* \\
\hline $\begin{array}{l}\text { Private Entwicklungshilfe } \\
\text { in \% des Bruttosozialprodukts }\end{array}$ & 0.06 & 0.06 & 0.06 & 0.06 & 0.07 & 0.07 & n.v.* \\
\hline $\begin{array}{l}\text { Öffentliche Entwicklungshilfe } \\
\text { in \% des Bruttosozialprodukts }\end{array}$ & 0.34 & 0.34 & 0.34 & 0.32 & 0.35 & 0.34 & 0.34 \\
\hline
\end{tabular}

Bemerkung: Die Beträge der privaten Entwicklungshilfe beziehen sich auf die eigenen Mittel der Hilfswerke, ohne öffentliche Beiträge. $\mathrm{p}$ : provisorische Beiträge.

${ }^{*}$ n.v.: noch nicht verfügbar.

Quelle: DEZA, April 2002. 